\title{
Dosimetry for Radiopharmaceutical Therapy: The European Perspective
}

\author{
Michael Lassmann ${ }^{1}$, Uta Eberlein ${ }^{1}$, Jonathan Gear ${ }^{2}$, Mark Konijnenberg ${ }^{3}$, and Jolanta Kunikowska ${ }^{4}$ \\ ${ }^{1}$ Department of Nuclear Medicine, University of Würzburg, Würzburg, Germany; ${ }^{2}$ Joint Department of Physics, Royal Marsden NHS \\ Foundation Trust and Institute of Cancer Research, London, United Kingdom; ${ }^{3}$ Department of Radiology and Nuclear Medicine, \\ Erasmus MC, Rotterdam, The Netherlands; and ${ }^{4}$ Nuclear Medicine Department, Medical University of Warsaw, Warsaw, Poland
}

\begin{abstract}
This review presents efforts in Europe over the last few years with respect to standardization of quantitative imaging and dosimetry and comprises the results of several European research projects on practices regarding radiopharmaceutical therapies (RPTs). Because the European Union has regulatory requirements concerning dosimetry in RPTs, the European Association of Nuclear Medicine released a position paper in 2021 on the use of dosimetry under these requirements. The importance of radiobiology for RPTs is elucidated in another position paper by the European Association of Nuclear Medicine. Furthermore, how dosimetry interacts with clinical requirements is described, with several clinical examples. In the future, more efforts need to be undertaken to increase teaching and standardization efforts and to incorporate radiobiology for further individualizing patient treatment, with the aim of improving the outcome and safety of RPTs.
\end{abstract}

Key Words: quantitative imaging; dosimetry; radiobiology

J Nucl Med 2021; 62:73S-79S

DOI: 10.2967/jnumed.121.262754

$\mathbf{T}$ he number of radiopharmaceutical therapies (RPTs) that have obtained marketing authorization in Europe has increased in recent years $\left({ }^{223} \mathrm{RaCl}_{2}\right.$ [Xofigo; Bayer] (1), ${ }^{177} \mathrm{Lu}$-oxodotreotide [Lutathera; Advanced Accelerator Applications] (2)), and several others are presently in late stages of clinical trials $\left({ }^{177} \mathrm{Lu}-\mathrm{PSMA}-617\right.$ (3), ${ }^{177}$ Lu-lilotomab (4)).

Dose-effect relationships after RPTs have been derived mostly from retrospective studies, the results of which have been nicely summarized in a review by Strigari et al. (5). For RPTs, prospective evidence with therapy prescription based on patient-specific dosimetry still needs to be obtained (6). Evidence demonstrating the superiority of dosimetry-guided prescription was provided in the DOSISPHERE trial on ${ }^{90} \mathrm{Y}$-microsphere therapy of liver cancer (7). This class of therapies is considered as treatment with a medical device and is therefore not considered further in this review of RPTs.

Quantitative imaging plays a major role in individualized treatment planning and posttherapeutic dose verification in RPTs. Sequential quantitative SPECT/CT measurements of therapeutically used radiopharmaceuticals permits determination of the

Received Jun. 28, 2021; revision accepted Oct. 13, 2021.

For correspondence or reprints, contact Uta Eberlein (eberlein_u@ukw.de). COPYRIGHT (C) 2021 by the Society of Nuclear Medicine and Molecular Imaging. spatial and temporal activity distribution in patients' organs or tissues $(8)$.

Several projects funded by the European Union's Horizon 2020 program provided additional input to the multistep approach needed for dosimetry in nuclear medicine (www.mrtdosimetryempir.eu, www.medirad-project.eu) $(9,10)$. Driven by the increasing number of therapeutic procedures, the European Association of Nuclear Medicine (EANM) dosimetry committee developed a guidance document for assessing uncertainties in absorbed dose calculations (11). As a European Council directive (12) requires pretherapeutic treatment planning and absorbed dose verification for RPTs (12), the EANM released a statement to help centers comply with the directive step by step, allowing for a change from the current practice to a patient-specific treatment ( 6 ) for the range of resources currently available across Europe. Because radiobiologic response is gaining increasing influence in clinical applications and in more fundamental research, the EANM also published a position paper on how best to integrate radiobiology in the world of nuclear medicine (13).

Consequently, the aim of this review is to summarize recent efforts in Europe for RPTs, with the aim of improving patient treatment by individualization based on dosimetry. RPTs, for this report, are defined as treatment with radiopharmaceuticals, in contrast to locoregional treatments with medical devices such as selective internal radiation therapy.

\section{MULTICENTER TRIALS INVOLVING STANDARDIZED QUANTITATIVE IMAGING AND DOSIMETRY}

In a recent review, Lassmann et al. summarized efforts to standardize quantitative imaging for dosimetry in major multicenter trials, mostly by European sites (14). Table 1 presents the setup and results for the most important SPECT/CT studies since 2018.

Wevrett et al. (15) reported on an intercomparison of quantitative imaging with ${ }^{177} \mathrm{Lu}$ in European hospitals using a shell sphere consisting of 2 isolated concentric spheres allowing the creation of a core filled with a high activity concentration, surrounded by a less active background shell (15). The authors concluded that reasonable uncertainties were reported by the participants; however, further research into the nature of the uncertainties should be done.

Peters et al. (16) evaluated the quantitative accuracy and intersystem variations for 4 Dutch centers by repeatedly scanning a cylindric phantom with 6 spheric inserts using standardized acquisition settings. The reconstructions were performed using vendor-specific algorithms and a vendor-neutral quantitative reconstruction for all systems. For each sphere, the authors 


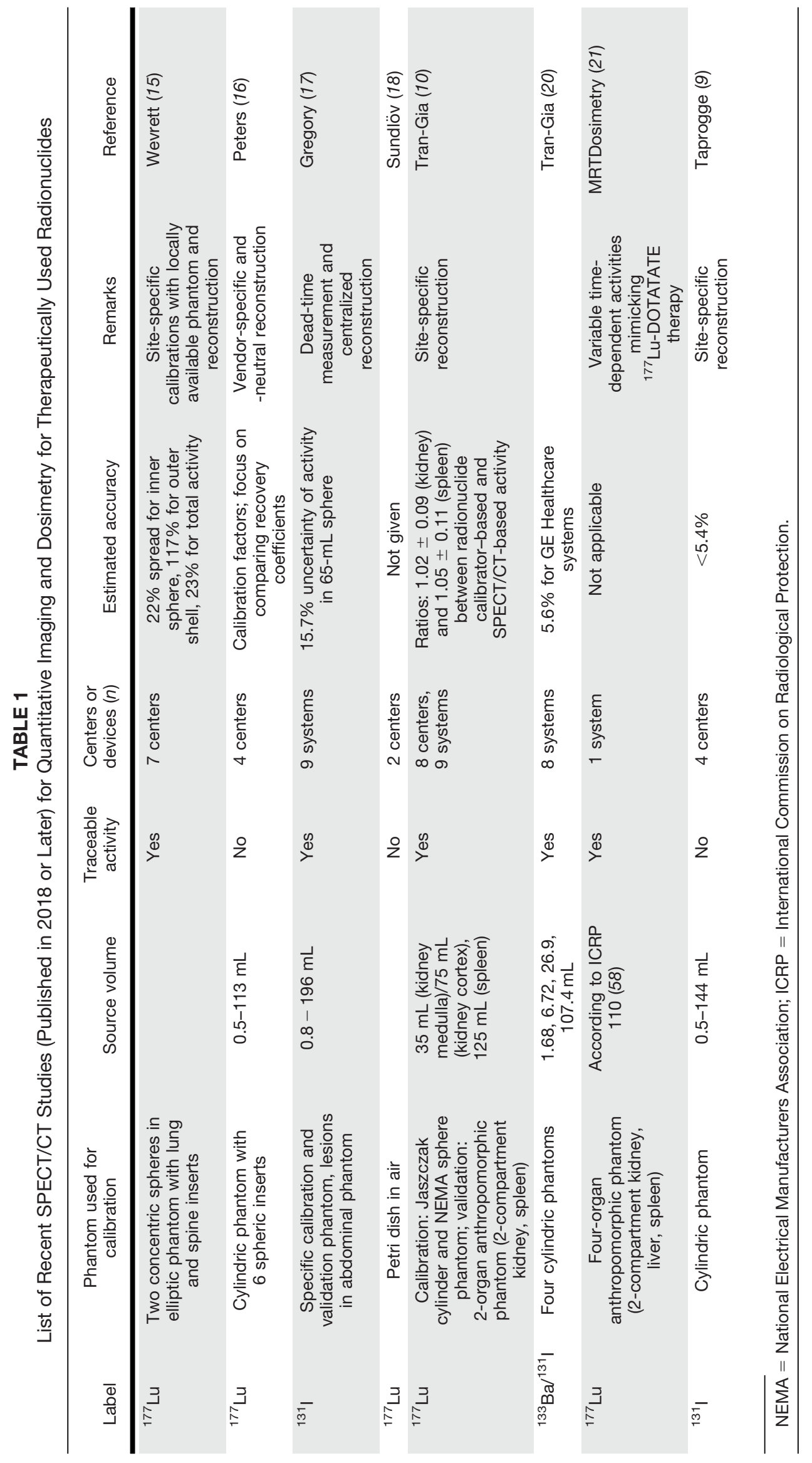

74S The Journal of Nuclear Medicine • Vol. 62 • No. 12 (Suppl. 3) • December 2021 
calculated mean and maximum recovery coefficients for 3 repeated measurements and defined the intersystem variations as the range of recovery coefficients over all systems. Overall, the authors concluded that eliminating the effects of system hardware and the use of standardized reconstruction algorithms is the key element for multicenter dosimetry and quantitative biomarker studies.

The first multicenter trial to investigate the role of ${ }^{123} \mathrm{I}$ and ${ }^{131} \mathrm{I}$ SPECT/CT-based tumor dosimetry in predicting response to radioiodine therapy was the SEL-I-METRY trial (17), which included a network of centers with consistent methods of radioiodine activity quantification (17). Image quantification was validated by imaging a 3-dimensionally printed phantom mimicking a patient's activity distribution. The errors in the validation of phantom activities were comparable to the measurement uncertainties derived from an uncertainty analysis. For example, the uncertainty for ${ }^{131} \mathrm{I}$ in a 5 -cm sphere was at $16 \%$ on average (17).

In a two-center Swedish study on the treatment of patients with neuroendocrine tumors, the aim was to determine the feasibility, safety, and efficacy of individualizing treatment with ${ }^{177} \mathrm{Lu}$-DOTATATE, based on renal dosimetry. The calibration of the systems used was derived from a planar scan or a SPECT/CT scan by using a thin layer of ${ }^{177} \mathrm{Lu}$ in a Petri dish placed in air, with the activity traceable to a standard laboratory (18).

Further efforts to standardize SPECT/CT calibration were undertaken in the joint European Metrology Research Project, MRTDosimetry (http://mrtdosimetry-empir.eu/), which terminated in mid-2019. The main goals of the project were to improve accuracy and metrologic traceability in the calculation of absorbed doses from time sequences of quantitative imaging measurements and to determine uncertainties in the relationship to the full dosimetry-related measurement chain from a primary standard to a range of commercial and noncommercial dosimetry calculation platforms. For this review, 3 subprojects are of interest. Regarding the first subproject, a study with ${ }^{177} \mathrm{Lu}$ by Tran-Gia et al. (10), the setup and results of a comparison exercise are reported. This study included 9 SPECT/CT systems with the same setup (system, acquisition, and reconstruction) for calibration, determination of the recovery coefficients for partial-volume correction, and a validation using a 3-dimensionally printed 2-organ phantom. The results were that similar combinations of imaging system and reconstruction led to image calibration factors that agreed within their respective uncertainties, provided the same software was used. Activity recovery still leads to uncertainties of up to $15 \%$. Accurate partial-volume correction still remains a challenge and is an unsolved problem, particularly at the voxel level (19).

The second subproject, an international quantitative SPECT/CT imaging comparison exercise, included 8 SPECT/CT systems and was set up to assess the applicability of ${ }^{133} \mathrm{Ba}$ sources as a surrogate for ${ }^{131} \mathrm{I}$ and to determine a cross-calibration factor (20). Cylinders of 4 different dimensions were fabricated with a 3-dimensional printing system and either were filled with solid ${ }^{133} \mathrm{Ba}$ (produced at 2 metrology institutions) or were left hollow, to be filled with liquid ${ }^{131} \mathrm{I}$ on site. Equivalent camera and reconstruction setups yielded comparable calibration factors. A cross-calibration factor between ${ }^{133} \mathrm{Ba}$ and

${ }^{131} \mathrm{I}$, which agreed with the ratio of the emission probabilities, was obtained, thus confirming that traceable solid ${ }^{133} \mathrm{Ba}$ sources are useful as surrogates for liquid ${ }^{131} \mathrm{I}$ in SPECT/CT calibrations (20).

In the third subproject, a series of SPECT/CT images was acquired of a 4-organ 3-dimensional phantom (left and right kidneys, liver, and spleen) and filled 6 times with varying ${ }^{177} \mathrm{Lu}$ activities mimicking 6 time points of a representative
${ }^{177}$ Lu-DOTATATE therapy (21), starting with a medulla-to-cortex ratio of $3: 1$. The late time point for scanning was $144 \mathrm{~h}$ after administration. To control the variability caused by camera and workstation setups, 6 sets of images were prepared (raw and reconstructed) to cover GE Healthcare, Siemens, and Hermes DICOM formats (21). In the near future, these data sets will be made publicly available for the testing and commissioning of dosimetry software.

Another European project, MEDIRAD (http://www.mediradproject.eu/), which started in 2017, aims to address the need to better understand and evaluate the health effects of low-dose ionizing radiation exposure from diagnostic and therapeutic imaging. In one of the work packages, necessary tools to establish, in a multicenter setting, the range of absorbed doses delivered to healthy organs of thyroid cancer patients undergoing thyroid ablation will be developed and implemented. The technical part of the project comprises standardization of quantitative imaging, as well as centralized dosimetry reading $(9)$.

The first results of these joint European efforts emphasize the need to define a standardized and reproducible calibration across sites for SPECT/CT quantitative imaging as a prerequisite for dosimetry in multicenter trials. Furthermore, for dosimetry, efforts in Europe include the OpenDose collaboration, which provides an open-access resource platform for available dosimetry data and tools to be used for nuclear medicine dosimetry (22).

\section{RECENT ACCOMPLISHMENTS OF THE EANM DOSIMETRY COMMITTEE}

The dosimetry task group, which subsequently became a committee of the EANM, was formed in 2001 (23) in response to an observed knowledge gap within the field. The committee founded the International Symposia on Radionuclide Therapy and Radiopharmaceutical Dosimetry. Four initial symposia were organized, between 2004 and 2011, coordinated jointly by the EANM radionuclide therapy committee in cooperation with the MIRD committee of the Society of Nuclear Medicine and Molecular Imaging (24). The success of these symposia translated into the regular Do.MoRe Track of the annual EANM congress, which continues to be a world-leading meeting attracting participation and attendees from around the globe, including America and Australia. In 2020, the track was reorganized to bring together all disciplines concerned with physics, dosimetry, and radiobiology and is now called the Cutting-Edge Science Track.

Teaching and dissemination of knowledge became a substantial component of the committee's activities, running courses on both basic and advanced dosimetry techniques. After the formation of the European School of Multimodality Imaging and Therapy, these courses were translated into a hands-on practical session covering the essentials for the implementation of dosimetry in nuclear medicine therapy. During the course, attendees are given the opportunity to work alongside dosimetry experts, processing raw scintigraphy data while learning the theory required to calculate an absorbed dose. In addition to the formal courses, committee members deliver numerous European School of Multimodality Imaging and Therapy webinars and lectures throughout Europe for national societies and other organizations. In 2013, a curriculum for education and training of medical physicists in nuclear medicine was developed in collaboration with the European Federation of Organizations in Medical Physics (25). Specific training courses for technologists have not been offered by the EANM; however, 
the dosimetry committee has been repeatedly involved in technologist teaching symposia on dosimetry during the annual EANM congresses.

The committee has contributed to several clinical EANM guidelines for both therapy and dosimetry (26-29) and continues to expand its series of standard operational procedures, including dosimetry procedures for ${ }^{131} \mathrm{I}$-MIBG treatment of neuroendocrine tumors, published in 2020 (30), and dosimetry in liver radioembolization with ${ }^{90} \mathrm{Y}$ microspheres (31). Similarly, a dosimetry guideline for ${ }^{177} \mathrm{Lu}$-labeled prostate-specific membrane antigen (PSMA)-targeting and somatostatin-receptor-targeting compounds is complete and shortly to be submitted. This document reviews current practice and dosimetry methods, complementing a previous publication (32).

Similar guidance has been produced to match the needs of the community. The guideline on good practices in clinical dosimetry reporting, published in 2011 (33), continues to provide essential advice for scientists who are preparing and submitting publications and reports containing data on internal dosimetry. Guidance describing a framework for modeling the uncertainty in the absorbed dose calculation was also provided to answer concerns over the accuracy and precision of clinical dosimetry (11). All these guidelines are available free of charge on the home page of the EANM (www. eanm.org).

In 2015, an EANM internal dosimetry task force was formed with the mandate of reporting on the current status and potential prospects of treatment planning for RPTs (34). The report evaluated whether dosimetry is feasible for the therapeutic procedures currently used, examined the evidence for absorbed dose-effect correlations, and speculated on how personalized treatment planning may be further developed $(34,35)$. The results of a survey by the task group (including representatives of the committee) describing variations in the practice of RPTs and implementation of dosimetry in Europe were also published (36).

Furthermore, the EANM supported the International Atomic Energy Agency initiative to write a soon-to-be-published handbook on dosimetry for RPTs in collaboration with the Society of Nuclear Medicine and Molecular Imaging, the American Association of Physicists in Medicine, and the European Federation of Organisations for Medical Physics. Members of the EANM dosimetry committee were also involved in writing the upcoming International Commission on Radiation Units and Measurements report 96 ("Dosimetry-Guided Radiopharmaceutical Therapy").

\section{COMPLIANCE WITH REGULATORY REQUIREMENTS IN EUROPE}

Regulatory requirements in the European Union differ slightly from those in the rest of the world. For the European Union, European Community Directive 2013/59/Euratom states in article 56 that "exposures of target volumes in nuclear medicine treatments shall be individually planned and their delivery appropriately verified" (12). Because the implementation of the directive differs in the European Union member states, the EANM considered it necessary to form a multidisciplinary working group to provide guidance on how to interpret article 56 of the directive with regard to RPTs (6). A potential discrepancy might arise with the European pharmaceutical regulations when personalized planning leads to a conflict between the approved prescription posology and the optimization principle of European Community directive 2013/59/ Euratom, as was summarized in the corresponding EANM position paper (6).
The paper proposes distinguishing between 3 levels of compliance with the optimization principle of the directive, inspired by the indication of levels in prescribing, recording, and reporting of absorbed doses in analogy to radiotherapy as described by International Commission on Radiation Units and Measurements report 91 (37). As stated in the position paper (6), level 1 is defined by administering the activity within $10 \%$ of the intended activity, typically according to the package insert or to the respective EANM guidelines, followed by verification of the therapy delivery, if applicable. Level 2, "Activity-Based Prescription and Patient-Specific Dosimetry," defines the need for dosimetry for nonstandardized treatments in the developmental phase or for approved radiopharmaceuticals being used off-label with significantly ( $>25 \%$ more than in the label) higher activities. This level implies recording and reporting of the absorbed dose to organs at risk and, optionally, the absorbed dose to treatment regions. Level 3, "Dosimetry-Guided Patient-Specific Prescription and Verification," is strongly encouraged by the EANM to foster research that eventually leads to treatment planning, whenever possible and relevant.

For many RPTs, the position paper provides examples of the minimum compliance level (6) for optimizing and standardizing patient-specific therapeutic practices in nuclear medicine in Europe. Because evidence of the superiority of therapy prescriptions based on patient-specific dosimetry has not been obtained yet for many therapeutic radiopharmaceuticals with a marketing authorization, the scheme was derived to help advance the field of RPTs. The scheme ensures that new therapies are introduced clinically and cost-effectively and that research for generating further evidence is stimulated. In addition, the authors state that a better understanding of radiobiology is key to the long-term improvement of RPTs (6).

Traditionally, safety and efficacy form the first aim in phase 1 and phase 2 clinical trials. This approach leads to generic knowledge about the therapeutic window for a radiopharmaceutical. Therefore, these trials provide an optimal opportunity to gather sufficient information on dose-response relationships for new RPTs (6).

\section{RADIOBIOLOGY}

In 2021, the EANM published a position paper on the role of radiobiology in nuclear medicine (13). For this paper, a group of EANM radiobiology, physics, and dosimetry experts summarized the main issues concerning radiobiology in nuclear medicine.

Extrapolation, to RPTs, of data obtained from the vast experience in radiobiology for external-beam radiation therapy or brachytherapy is complex because of differences in absorbed dose rates and spatial and temporal dose distributions. As a result, irradiated organs and tissues respond differently in RPTs (38-40), and the condition of RPT patients with metastatic disease diverges considerably from external-beam radiation therapy patients with single tumors. DNA damage induction and repair will strongly differ from the external-beam radiation therapy experience because of the comparatively low dose rates varying over time with physical decay and kinetic clearance, such as in patients after prostate cancer RPT $(41,42)$. Repair of sublethal DNA damage proceeds in parallel to the absorbed dose delivery, and this effect has led to a higher threshold in the absorbed dose, inducing late kidney damage after ${ }^{90} \mathrm{Y}$ peptide therapy in comparison to the well-established external-beam radiation therapy threshold dose (43). Consequently, 
there is a need to generate and apply more radiobiologic knowledge specific to nuclear medicine diagnostic and therapeutic procedures.

In the EANM position paper, the authors provided an example concerning ${ }^{177} \mathrm{Lu}$-DOTATATE therapy with advanced, progressive, somatostatin receptor subtype 2-positive midgut neuroendocrine tumors, the patient group that was studied in the NETTER-1 phase 3 trial (44). Further optimization of ${ }^{177}$ Lu-DOTATATE therapy while keeping toxicity low may include improved personalized dosimetry (45) in conjunction with a deep biologic evaluation of superior radionuclides, improved somatostatin receptor subtype 2 ligands, increased somatostatin receptor subtype 2 levels, the role of tumor microenvironment, and combinations with immunotherapy, targeted therapy, or DNA-modulating agents, as well as predictive markers for improved patient selection and treatment follow-up (46-50).

The position of the EANM is that radiobiology will contribute to the optimization of RPTs to ensure that they are effective and safe for each individual patient (13). It is expected that a better understanding of radiobiologic parameters will enhance the capabilities of new and existing nuclear medicine applications. There is a need to better define the dose-effect relationships of systemic ionizing radiation for tumors and for normal tissue. To achieve this goal, the EANM recommends a strong link between all disciplines involved (radiochemists, radiopharmacists, radiobiologists, medical physicists, and physicians) (13).

\section{DOSIMETRY AND THE INTERACTION WITH CLINICAL REQUIREMENTS}

Recent RPTs with marketing authorization, such as ${ }^{223} \mathrm{RaCl}_{2}$ or ${ }^{177} \mathrm{Lu}$-DOTATATE therapy, investigated dosimetry during initial phase I and II trials. With other therapies, such as ${ }^{131} \mathrm{I}-\mathrm{NaI}, 80 \mathrm{y}$ of experience has shown that treatment of patients with fixed activities or based on disease, thyroid uptake, and volume is safe, with limited side effects.

${ }^{131}$ I-MIBG has been used as an effective salvage therapy over many decades for pheochromocytoma, neuroblastoma, medullary thyroid carcinoma, and selected cases of neuroendocrine. Hemotoxicity may be observed in some patients but can be well tolerated and controlled with stem cell harvest if necessary.

${ }^{177}$ Lu-DOTATATE therapy for neuroendocrine patients, with $30 \mathrm{y}$ of experience, is based on the Rotterdam protocol with the 4 injections of $7.4 \mathrm{GBq}$ per cycle $(29.6 \mathrm{GBq}$ in total) $(2,44)$. For peptide radionuclide therapy, the main side effect is nephrotoxicity, with the kidney as the dose-limiting organ. Using nephroprotection with amino acid infusion, renal toxicity is reduced to occasional cases. A dosimetry-tailored activity escalation study on 200 patients receiving $22.2-74 \mathrm{GBq}(51)$ and a second study on 74 patients receiving $14.8-37.8 \mathrm{GBq}$ (52) showed renal toxicity grades 3-4 in only 1 patient. Salvage treatments administering up to 4 additional peptide radionuclide therapy cycles, and cumulative activities of up to $60.5 \mathrm{GBq}$ for ${ }^{177} \mathrm{Lu}$-DOTATATE (53) or up to $30.7 \mathrm{GBq}$ for tandem ${ }^{90} \mathrm{Y} /{ }^{177} \mathrm{Lu}$-DOTATATE (54), did not show any increase in kidney or bone marrow-related side effects.

The latest development in ${ }^{177} \mathrm{Lu}-\mathrm{PSMA}$ therapy for prostate cancer patients showed a very good clinical response, with limited side effects. The results of the VISION phase III study found a 4-mo gain in life expectancy and a 5-mo delay in disease progression in the $6 \times 7.4-\mathrm{GBq}$ treatment group, compared with the standard of care (3). Most side effects were mild, of grade 1-2, and comprised bone marrow suppression (47\%), dry mouth $(39 \%)$, hepatotoxicity (10\%), and renal effects (9\%) (3). Adverse events of at least grade 3 were higher for ${ }^{177}$ Lu-PSMA therapy than for the standard of care $(52.7 \%$ vs. $38 \%)$ but did not, however, have an impact on quality of life (3).

From a clinical perspective, the most important issue regarding treatment optimization is always patient safety. However, preservation and improvement of quality of life are no less significant.

Treatment optimization and personalization through individual planning of the absorbed doses delivered to target organs, taking into account the absorbed doses delivered to nontarget organs, is a challenge. When optimizing treatment procedures, we need to consider that the main goal is to help and treat the patient. The object of our research is, in most cases, an oncologic patient with an oftenpoor clinical condition and progressive disease. Swift and efficient treatment is paramount, and for this reason dosimetry-guided prescriptions should not delay the start of treatment procedures.

Most dosimetry approaches require quantitative imaging, ideally up to 4 or 6 time points. Depending on the imaging modality, these could take up to 30-60 min. For many clinical centers, this may be difficult to achieve. Access to SPECT/CT systems may be limited in busy centers occupied with other daily imaging, and the patients' condition and quality of life should be considered. For patients with a poor performance status, simplified personalized dosimetry regimens could become an important asset. Dosimetry based on single-time-point acquisitions, particularly using only 1 SPECT/CT scan, are now on the rise and could offer a compromise between the accuracy and resources needed for dosimetry (55). Such dosimetry could also be perfectly connected to the development of artificial intelligence methods to improve the dosimetry-guided treatment planning $(56,57)$.

Several areas have been identified in which dosimetry plays an important role and is highly desired: RPT in children, radiopharmaceuticals under clinical development, and off-label use of radiopharmaceuticals with administrations of activity that are significantly higher $(\geq 25 \%)$ than the recommended activity, including the total activity accumulated over all cycles and treatments (6). In daily clinical practice, dosimetry studies could also be considered in selected patients with risk factors.

As has been discussed in the EANM position papers $(6,13)$, a better understanding of therapy dosimetry, that is, how much and where the energy is delivered, and radiobiology, that is, radiationrelated processes in tissues, are keys to the long-term improvement of our treatments.

\section{CONCLUSION}

Today, standardization of quantitative imaging and dosimetry between laboratories is feasible. However, care has to be taken to minimize the variability in image acquisition and reconstruction. In the next few years, further individualization of patient treatment will be needed, as well as greater effort to increase teaching and standardization and to incorporate radiobiology — all with an aim of improving the outcome and safety of patients undergoing RPTS.

\section{DISCLOSURE}

No potential conflict of interest relevant to this article was reported.

\section{REFERENCES}

1. Poeppel TD, Handkiewicz-Junak D, Andreeff M, et al. EANM guideline for radionuclide therapy with radium-223 of metastatic castration-resistant prostate cancer. Eur J Nucl Med Mol Imaging. 2018;45:824-845. 
2. Bodei L, Mueller-Brand J, Baum RP, et al. The joint IAEA, EANM, and SNMMI practical guidance on peptide receptor radionuclide therapy (PRRNT) in neuroendocrine tumours. Eur J Nucl Med Mol Imaging. 2013;40:800-816.

3. Sartor O, de Bono J, Chi KN, et al. Lutetium-177-PSMA-617 for metastatic castration-resistant prostate cancer. $N$ Engl J Med. 2021;385:1091-1103.

4. Kolstad A, Illidge T, Bolstad N, et al. Phase $1 / 2$ a study of ${ }^{177}$ Lu-lilotomab satetraxetan in relapsed/refractory indolent non-Hodgkin lymphoma. Blood Adv. 2020;4: 4091-4101.

5. Strigari L, Konijnenberg M, Chiesa C, et al. The evidence base for the use of internal dosimetry in the clinical practice of molecular radiotherapy. Eur J Nucl Med Mol Imaging. 2014;41:1976-1988.

6. Konijnenberg M, Herrmann K, Kobe C, et al. EANM position paper on article 56 of the Council Directive 2013/59/Euratom (basic safety standards) for nuclear medicine therapy. Eur J Nucl Med Mol Imaging. 2021;48:67-72.

7. Garin E, Tselikas L, Guiu B, et al. Personalised versus standard dosimetry approach of selective internal radiation therapy in patients with locally advanced hepatocellular carcinoma (DOSISPHERE-01): a randomised, multicentre, openlabel phase 2 trial. Lancet Gastroenterol Hepatol. 2021;6:17-29.

8. Flux G, Bardies M, Monsieurs M, Savolainen S, Strands SE, Lassmann M. The impact of PET and SPECT on dosimetry for targeted radionuclide therapy. Z Med Phys. 2006;16:47-59.

9. Taprogge J, Leek F, Schurrat T, et al. Setting up a quantitative SPECT imaging network for a European multi-centre dosimetry study of radioiodine treatment for thyroid cancer as part of the MEDIRAD project. EJNMMI Phys. 2020;7:61.

10. Tran-Gia J, Denis-Bacelar AM, Ferreira KM, et al. A multicentre and multinational evaluation of the accuracy of quantitative Lu-177 SPECT/CT imaging performed within the MRTDosimetry project. EJNMMI Phys. 2021;8:55.

11. Gear JI, Cox MG, Gustafsson J, et al. EANM practical guidance on uncertainty analysis for molecular radiotherapy absorbed dose calculations. Eur J Nucl Med Mol Imaging. 2018;45:2456-2474.

12. Council of the European Union. European Council directive 2013/59/Euratom on basic safety standards for protection against the dangers arising from exposure to ionising radiation and repealing directives 89/618/Euratom, 90/641/Euratom, 96/29/Euratom, 97/43/Euratom and 2003/122/Euratom. Off J Eur Union. 2014; L13:1-73.

13. Aerts A, Eberlein U, Holm S, et al. EANM position paper on the role of radiobiology in nuclear medicine. Eur J Nucl Med Mol Imaging. 2021;48:3365-3377.

14. Lassmann M, Eberlein U, Tran-Gia J. Multicentre trials on standardised quantitative imaging and dosimetry for radionuclide therapies. Clin Oncol (R Coll Radiol). 2021;33:125-130.

15. Wevrett J, Fenwick A, Scuffham J, et al. Inter-comparison of quantitative imaging of lutetium-177 $\left({ }^{177} \mathrm{Lu}\right)$ in European hospitals. EJNMMI Phys. 2018;5:17.

16. Peters SMB, Meyer Viol SL, van der Werf NR, et al. Variability in lutetium-177 SPECT quantification between different state-of-the-art SPECT/CT systems. EJNMMI Phys. 2020;7:9.

17. Gregory RA, Murray I, Gear J, et al. Standardised quantitative radioiodine SPECT/ CT imaging for multicentre dosimetry trials in molecular radiotherapy. Phys Med Biol. 2019;64:245013.

18. Sundlöv A, Sjogreen-Gleisner K, Svensson J, et al. Individualised ${ }^{177}$ Lu-DOTATATE treatment of neuroendocrine tumours based on kidney dosimetry. Eur J Nucl Med Mol Imaging. 2017;44:1480-1489.

19. Tran-Gia J, Salas-Ramirez M, Lassmann M. What you see is not what you get: on the accuracy of voxel-based dosimetry in molecular radiotherapy. J Nucl Med. 2020;61:1178-1186.

20. Tran-Gia J, Robinson AP, Bobin C, et al. An international quantitative SPECT/CT imaging exercise for assessment of Ba-133 as surrogate for I-131 [abstract]. Eur J Nucl Med Mol Imaging. 2020;47(suppl):S448-S449.

21. Final publishable report. http://mrtdosimetry-empir.eu/wp-content/uploads/2019/ 08/15HLT06_Publishable_Report_M36_Final.pdf. MRTDosimetry website. Published June 1, 2016. Accessed October 18, 2021

22. Chauvin M, Borys D, Botta F, et al. OpenDose: open-access resource for nuclear medicine dosimetry. J Nucl Med. 2020;61:1514-1519.

23. Flux GD, Bardies M, Lassmann M. Biting the magic bullet: celebrating a decade of the EANM Dosimetry Committee. Eur J Nucl Med Mol Imaging. 2014;41:1-3.

24. Chiesa C, Luster M, Lassmann M. 4th international symposium on targeted radiotherapy and dosimetry (ISTARD): best ranked abstract publication. Q J Nucl Med Mol Imaging. 2012;56:485-486.

25. Del Guerra A, Bardies M, Belcari N, et al. Curriculum for education and training of medical physicists in nuclear medicine: recommendations from the EANM Physics Committee, the EANM Dosimetry Committee and EFOMP. Phys Med. 2013;29:139-162.

26. Giammarile F, Bodei L, Chiesa C, et al. EANM procedure guideline for the treatment of liver cancer and liver metastases with intra-arterial radioactive compounds. Eur J Nucl Med Mol Imaging. 2011;38:1393-1406.
27. Hindorf C, Glatting G, Chiesa C, Linden O, Flux G, Committee ED. EANM Dosimetry Committee guidelines for bone marrow and whole-body dosimetry. Eur J Nucl Med Mol Imaging. 2010;37:1238-1250.

28. Kratochwil C, Fendler WP, Eiber M, et al. EANM procedure guidelines for radionuclide therapy with Lu-177-labelled PSMA-ligands (Lu-177-PSMA-RLT). Eur J Nucl Med Mol Imaging. 2019;46:2536-2544.

29. Lassmann M, Hänscheid H, Chiesa C, et al. EANM Dosimetry Committee series on standard operational procedures for pre-therapeutic dosimetry I: blood and bone marrow dosimetry in differentiated thyroid cancer therapy. Eur J Nucl Med Mol Imaging. 2008;35:1405-1412.

30. Gear J, Chiesa C, Lassmann M, et al. EANM Dosimetry Committee series on standard operational procedures for internal dosimetry for ${ }^{131} \mathrm{I}$ mIBG treatment of neuroendocrine tumours. EJNMMI Phys. 2020;7:15.

31. Chiesa C, Sjogreen-Gleisner K, Walrand S, et al. EANM Dosimetry Committee Series on Standard Operational Procedures: a unified methodology for ${ }^{99 \mathrm{~m}} \mathrm{Tc}-\mathrm{MAA}$ pre- and ${ }^{90} \mathrm{Y}$ peri-therapy dosimetry in liver embolization with ${ }^{90} \mathrm{Y}$ microspheres. EJNMMI Phys. In press. https://doi.org/10.1186/s40658-021-00394-3.

32. Ljungberg M, Celler A, Konijnenberg MW, et al. MIRD pamphlet no. 26: joint EANM/MIRD guidelines for quantitative ${ }^{177} \mathrm{Lu}$ SPECT applied for dosimetry of radiopharmaceutical therapy. $J$ Nucl Med. 2016;57:151-162.

33. Lassmann M, Chiesa C, Flux G, Bardies M, Committee ED. EANM Dosimetry Committee guidance document: good practice of clinical dosimetry reporting. Eur J Nucl Med Mol Imaging. 2011;38:192-200.

34. Stokke C, Gabina PM, Solny P, et al. Dosimetry-based treatment planning for molecular radiotherapy: a summary of the 2017 report from the Internal Dosimetry Task Force. EJNMMI Phys. 2017;4:27.

35. Internal dosimetry task force report on: treatment planning for molecular radiotherapy - potential and prospects. European Association of Nuclear Medicine website. https://www.eanm.org/content-eanm/uploads/documents/EANM_2017_iDTFReport_online.pdf. Accessed October 18, 2021.

36. Sjögreen Gleisner K, Spezi E, Solny P, et al. Variations in the practice of molecular radiotherapy and implementation of dosimetry: results from a European survey. EJNMMI Phys. 2017;4:28.

37. Report 91. J ICRU. 2014;14:1-160.

38. Pouget JP, Lozza C, Deshayes E, Boudousq V, Navarro-Teulon I. Introduction to radiobiology of targeted radionuclide therapy. Front Med (Lausanne). 2015;2:12.

39. Sgouros G, Bodei L, McDevitt MR, Nedrow JR. Radiopharmaceutical therapy in cancer: clinical advances and challenges. Nat Rev Drug Discov. 2020;19:589-608.

40. Morris ZS, Wang AZ, Knox SJ. The radiobiology of radiopharmaceuticals. Semin Radiat Oncol. 2021;31:20-27.

41. Schumann S, Scherthan H, Lapa C, et al. DNA damage in blood leucocytes of prostate cancer patients during therapy with ${ }^{177}$ Lu-PSMA. Eur J Nucl Med Mol Imaging. 2019;46:1723-1732.

42. Schumann S, Eberlein U, Lapa C, et al. $\alpha$-particle-induced DNA damage tracks in peripheral blood mononuclear cells of $\left[{ }^{223} \mathrm{Ra}\right] \mathrm{RaCl}_{2}$-treated prostate cancer patients. Eur J Nucl Med Mol Imaging. 2021;48:2761-2770.

43. Barone R, Borson-Chazot F, Valkema R, et al. Patient-specific dosimetry in predicting renal toxicity with ${ }^{90} \mathrm{Y}$-DOTATOC: relevance of kidney volume and dose rate in finding a dose-effect relationship. J Nucl Med. 2005;46(suppl 1): 99S-106S.

44. Strosberg J, El-Haddad G, Wolin E, et al. Phase 3 trial of ${ }^{177}$ Lu-dotatate for midgut neuroendocrine tumors. N Engl J Med. 2017;376:125-135.

45. Sundlöv A, Sjogreen-Gleisner K. Peptide receptor radionuclide therapy: prospects for personalised treatment. Clin Oncol (R Coll Radiol). 2021;33:92-97.

46. Hofving T, Sandblom V, Arvidsson Y, et al. ${ }^{177}$ Lu-octreotate therapy for neuroendocrine tumours is enhanced by Hsp90 inhibition. Endocr Relat Cancer. 2019;26: $437-449$.

47. Brabander T, Nonnekens J, Hofland J. The next generation of peptide receptor radionuclide therapy. Endocr Relat Cancer. 2019;26:C7-C11.

48. Feijtel D, de Jong M, Nonnekens J. Peptide receptor radionuclide therapy: looking back, looking forward. Curr Top Med Chem. 2020;20:2959-2969.

49. Bodei L, Kidd MS, Singh A, et al. PRRT genomic signature in blood for prediction of ${ }^{177}$ Lu-octreotate efficacy. Eur J Nucl Med Mol Imaging. 2018;45: 1155-1169.

50. Bodei L, Kidd MS, Singh A, et al. PRRT neuroendocrine tumor response monitored using circulating transcript analysis: the NETest. Eur J Nucl Med Mol Imaging. 2020;47:895-906.

51. Garske-Román U, Sandstrom M, Fross Baron K, et al. Prospective observational study of ${ }^{177}$ Lu-DOTA-octreotate therapy in 200 patients with advanced metastasized neuroendocrine tumours (NETs): feasibility and impact of a dosimetryguided study protocol on outcome and toxicity. Eur J Nucl Med Mol Imaging. 2018;45:970-988. 
52. Sabet A, Ezziddin K, Pape UF, et al. Accurate assessment of long-term nephrotoxicity after peptide receptor radionuclide therapy with ${ }^{177}$ Lu-octreotate. Eur J Nucl Med Mol Imaging. 2014;41:505-510.

53. van der Zwan WA, Brabander T, Kam BLR, et al. Salvage peptide receptor radionuclide therapy with $\left[{ }^{177} \mathrm{Lu}-\mathrm{DOTA}, \mathrm{Tyr}^{3}\right]$ octreotate in patients with bronchial and gastroenteropancreatic neuroendocrine tumours. Eur J Nucl Med Mol Imaging. 2019;46:704-717.

54. Zemczak A, Gut P, Pawlak D, et al. The safety and efficacy of the repeated PRRT with $\left[{ }^{90} \mathrm{Y}\right] \mathrm{Y} /\left[{ }^{177} \mathrm{Lu}\right] \mathrm{Lu}-\mathrm{DOTATATE}$ in patients with NET. Int J Endocrinol. 2021; 2021:6615511.
55. Hänscheid H, Lassmann M. Will SPECT/CT cameras soon be able to display absorbed doses? Dosimetry from single activity concentration measurements. J Nucl Med. 2020;61:1028-1029.

56. Götz TI, Schmidkonz C, Chen S, Al-Baddai S, Kuwert T, Lang EW. A deep learning approach to radiation dose estimation. Phys Med Biol. 2020;65:035007.

57. Rydén T, Van Essen M, Marin I, Svensson J, Bernhardt P. Deep-learning generation of synthetic intermediate projections improves ${ }^{177} \mathrm{Lu}$ SPECT images reconstructed with sparsely acquired projections. J Nucl Med. 2021;62:528-535.

58. Menzel HG, Clement C, DeLuca P. ICRP publication 110: adult reference computational phantoms. Ann ICRP. 2009;39:1-164. 